\title{
Therapy of an incomplete spinal cord injury by intrathecal injection of EPO and subcutaneous injection of EPO, vitamin C and G-CSF
}

\author{
This article was published in the following Dove Press journal: \\ Therapeutics and Clinical Risk Management \\ Number of times this article has been viewed
}

\begin{abstract}
Augustinus Bader'
Martin Reinhardt ${ }^{\prime}$

Achim Beuthe ${ }^{2}$

Klaus Röhl ${ }^{2}$

Shibashish Giri ${ }^{1,3}$

'Department of Cell Techniques and Applied Stem Cell Biology, Centre for Biotechnology and Biomedicine, Medical faculty of University of Leipzig, Leipzig, ${ }^{2}$ Department of Orthopedic Surgery, BG clinics Bergmannstrost Halle, ${ }^{3}$ Department of Plastic and Hand Surgery, University Hospital Rechts der Isar, Munich Technical University, Munich, Germany
\end{abstract}

Correspondence: Augustinus Bader; Shibashish Giri

Department of Cell Techniques and Applied Stem Cell Biology, Center for Biotechnology and Biomedicine, Medical faculty of University of Leipzig, Deutscher Platz 5, D-04, 103 Leipzig, Germany

Tel +4934 I973 I353

Fax +4934 $1973 \quad 1329$

Email augustinus.bader@bbz.uni-leipzig.de; shibashish.giri@bbz.uni-leipzig.de

\begin{abstract}
Spinal cord injury is a rare disease with an incidence about 40 cases per million population in the USA. The most common reasons are traffic accidents, falls, violence and sports. A 53-year-old male patient presented with an incomplete tetraparesis as a result of a spinal cord injury after the accident. It was not possible to treat him with steroids because he was out of the therapeutic time period of 8 hours when he presented to the hospital. The main problem of spinal cord injuries is the secondary injury caused by inflammation and swelling of the spinal cord. To avoid this, the patient was experimentally treated with erythropoietin (EPO) intrathecal and EPO, granulocyte-colony-stimulating factor and vitamin $\mathrm{C}$ subcutaneous after his initial spinal cord relief surgery. These drugs might be able to relieve this secondary reaction but were never applied for this indication in human before. This study shows that it could be a promising treatment for spinal cord injuries with potential therapeutic benefits.
\end{abstract}

Keywords: EPO, G-CSF, spinal cord injury, vitamin C

\section{Introduction}

The treatment of a spinal cord injury (SCI) in combination with fractures of the vertebrae depends on the stability of the spine. Spinal injuries with neurological symptoms should be treated immediately to give relief to the spinal cord and avoid secondary injury due to inflammation, edema or insufficient blood supply. An early operative treatment within the first 24 hours after the injury significantly improves the neurological outcome of the patients. ${ }^{1}$ In lesions of the lower cervical spine, a ventral stabilization with a plate is sufficient. A dorsal fixation is often only necessary in category $\mathrm{C}$ fractures. A conservative therapy, using a cervical collar, is possible if the fracture/injury of the spine is stable and without neurological symptoms.

It is well known that the majority of the SCIs are caused secondarily by multifactorial, interrelated processes, including ischemia, reactive oxygen lipid peroxidation, edema, generation of free radicals and excitotoxicity. The National Acute Spinal Cord Injury Study II/III studies come to the result that an initial treatment with methylprednisolone within the first 8 hours after an injury for up to 48 hours can improve the neurological outcome independent of surgical treatment. ${ }^{2}$ The patients in this trial received an initial bolus of $30 \mathrm{mg}$ methylprednisolone per $\mathrm{kg}$ bodyweight and a maintenance dose of $5.4 \mathrm{mg}$ per $\mathrm{kg}$ bodyweight. This therapy is/was not indicated when applied 8 hours after the event of injury. This treatment was criticized in the 
last years because of the massive possible side effects (eg, infections) and the missing clinical evidence. Other therapy goals are the prevention of a spinal shock and the immobilization of the patient.

\section{Case report}

A 53-year-old male patient presented with a tetraparesis after the accident. He first woke up in a hospital after the accident. After 1 day, he was brought to a hospital in Germany for diagnosis and treatment.

The patient had a reduced muscle strength on examination. It is shown in Table 1. The muscles of the right side of his body were weaker than the left side. Furthermore, the right hip flexors were not affected as much as the muscles in the other segments below C5. The anal sphincter was floppy but could be activated to a minor degree from the right side. Clinical testing on the muscle strength showed that the patient had spared muscle movement below the level of the injury and could not move against gravity on one half of his body. The other half was not effected in this way. In conclusion, the right part of his body has to be classified as American Spinal Injury Association (ASIA) C, while the left part of his body can be classified as ASIA D.

The patient has to be checked for injuries of the vertebrae, the spinal cord and the soft tissue of his dorsum. Fractures can be assessed using X-ray or computed tomography (CT) of the spine. The CT imaging should be preferred if available because it is not possible to display the complete spine using X-ray. Especially the cervicothoracic junction is often superimposed by the shoulders. The biggest advantage of the $\mathrm{CT}$ is the possibility to create multiplanar reconstructions of different planes of the spine. Magnetic resonance imaging (MRI) is necessary to evaluate whether the patient

Table I Functional grading of the key muscles before surgery

\begin{tabular}{llll}
\hline Segment & Key muscles & Right & Left \\
\hline C5 & Elbow flexors & $5 / 5$ & $5 / 5$ \\
C6 & Wrist extensors & $0 / 5$ & $3 / 5$ \\
C7 & Elbow extensors & $1 / 5$ & $3 / 5$ \\
C8 & Finger flexors (distal phalanx of middle finger) & $0 / 5$ & $4 / 5$ \\
ThI & Finger abductors (little finger) & $0 / 5$ & $3 / 5$ \\
L2 & Hip flexors & $3 / 5$ & $5 / 5$ \\
L3 & Knee extensors & $0 / 5$ & $4 / 5$ \\
L4 & Ankle dorsiflexors & $0 / 5$ & $4 / 5$ \\
L5 & Long toe extensors & $0 / 5$ & $4 / 5$ \\
S1 & Ankle plantar flexors & $0 / 5$ & $4 / 5$ \\
\hline
\end{tabular}

Notes: Muscle function grading: 0 , total paralysis; I, palpable contraction; 2, active movement with full range of motion (ROM) with gravity eliminated; 3 , active movement with full ROM against gravity; 4, active movement with full ROM against moderate resistance; 5 , active movement with full ROM against full resistance.

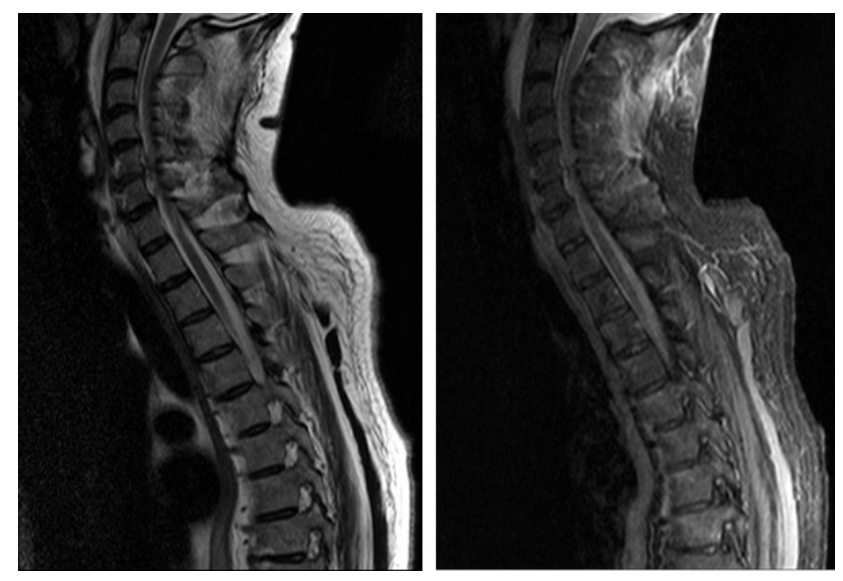

Figure I Magnetic resonance images of soft tissue damage and the spinal cord injury.

has a direct or indirect (swelling/edema/bleeding) injury of the spinal cord or soft tissue injury. There are also patients who have a neurological deficit but no radiographic evidence for a trauma. This is called SCIWORA (Spinal Cord Injury Without Radiographic Abnormalities).

The patient initially received a CT, which showed an avulsion of the anterior inferior margin of the 6th cervical vertebra. Additionally there were fractures of the transverse processes of the 6 th and 7 th cervical vertebra, the 1 st thoracic vertebra and rib fractures from the 4 th to the 8 th rib on the left side. To evaluate the soft tissue damage and the injury of the spinal cord, he also underwent an MRI. A discoligamentous lesion between $\mathrm{C} 5$ and $\mathrm{C} 6$, as well as a swelling of the spinal cord was detected and is shown in Figure 1.

The patient received no methylprednisolone because he arrived more than 8 hours after the injury happened. The intervertebral disc between the cervical vertebras 5 and 6 was removed and the vertebras were joined. During this surgery, a suprapubic fistula of the bladder was applied to allow the urination.

To reduce the influence of the secondary injury the patient received an experimental therapy with an initial intrathecal application of erythropoietin (EPO). From the first postsurgery day until the 58th postsurgery he was treated with subcutaneous injections of EPO, granulocyte colony-stimulating factor (G-CSF) and vitamin C every 3-5 days and regular control of his laboratory values.

\section{Results}

During the next weeks, the patient regained motoric abilities to his limbs. This progress was monitored every week using the functional grading scale for muscles and is shown in Tables 2-7. Two and a half months after his surgery he could 
Table 2 Functional grading of the key muscles 10 days postsurgery

\begin{tabular}{llll}
\hline Segment & Key muscles & Right & Left \\
\hline C5 & Elbow flexors & $5 / 5$ & $5 / 5$ \\
C6 & Wrist extensors & $2 / 5$ & $3 / 5$ \\
C7 & Elbow extensors & $1 / 5$ & $3 / 5$ \\
C8 & Finger flexors (distal phalanx of middle finger) & $0 / 5$ & $4 / 5$ \\
ThI & Finger abductors (little finger) & $0 / 5$ & $3 / 5$ \\
L2 & Hip flexors & $3 / 5$ & $5 / 5$ \\
L3 & Knee extensors & $2 / 5$ & $4 / 5$ \\
L4 & Ankle dorsiflexors & $0 / 5$ & $4 / 5$ \\
L5 & Long toe extensors & $0 / 5$ & $4 / 5$ \\
SI & Ankle plantar flexors & $0 / 5$ & $4 / 5$ \\
\hline
\end{tabular}

Notes: Muscle function grading: 0 , total paralysis; I, palpable contraction; 2 , active movement with full range of motion (ROM) with gravity eliminated; 3, active movement with full ROM against gravity; 4 , active movement with full ROM against moderate resistance; 5 , active movement with full ROM against full resistance.

empty his bladder via naturalis with amounts of residual urine of 200-300 mL, which required additional catheterization. On his release date 3 months after his accident he had full control over his anal sphincter. He had to intermittently catheterize his bladder two to three times a day. He nearly regained his complete motoric function but was limited in his stamina, and is shown in Table 8 . He was able to drive a car by himself.

The pathophysiology of secondary injury in the injured spinal cord was well described elsewhere. ${ }^{3}$ The $\mathrm{SCI}$ is one of the most devastating injuries, contributing to functional shortfalls including hind limb function. It often occurs in spine trauma caused by a motor vehicle accident. SCIs may be complete or incomplete injury. We present here the incomplete injury of a 53-year-old patient with a tetraparesis after the accident. The current treatment does not repair the spine trauma in the spinal cord. Instead, it mitigates the secondary damage following the spinal trauma. EPO is a hormone that controls the production of red blood cells and also a signaling molecule for erythrocyte precursor cells. High levels of

Table 3 Functional grading of the key muscles I 3 days postsurgery

\begin{tabular}{llll}
\hline Segment & Key muscles & Right & Left \\
\hline C5 & Elbow flexors & $5 / 5$ & $5 / 5$ \\
C6 & Wrist extensors & $2+/ 5$ & $4 / 5$ \\
C7 & Elbow extensors & $1 / 5$ & $4 / 5$ \\
C8 & Finger flexors (distal phalanx of middle finger) & $0 / 5$ & $4 / 5$ \\
ThI & Finger abductors (little finger) & $0 / 5$ & $3 / 5$ \\
L2 & Hip flexors & $3 / 5$ & $5 / 5$ \\
L3 & Knee extensors & $2+/ 5$ & $4 / 5$ \\
L4 & Ankle dorsiflexors & $1 / 5$ & $4 / 5$ \\
L5 & Long toe extensors & $1 / 5$ & $4 / 5$ \\
S1 & Ankle plantar flexors & $0 / 5$ & $4 / 5$ \\
\hline
\end{tabular}

Notes: Muscle function grading: 0 , total paralysis; I, palpable contraction; 2 , active movement with full range of motion (ROM) with gravity eliminated; 3 , active movement with full ROM against gravity; 4, active movement with full ROM against moderate resistance; 5 , active movement with full ROM against full resistance.
Table 4 Functional grading of the key muscles 16 days postsurgery

\begin{tabular}{llll}
\hline Segment & Key muscles & Right & Left \\
\hline C5 & Elbow flexors & $5 / 5$ & $5 / 5$ \\
C6 & Wrist extensors & $2+/ 5$ & $4 / 5$ \\
C7 & Elbow extensors & $2 / 5$ & $4 / 5$ \\
C8 & Finger flexors (distal phalanx of middle finger) & $0 / 5$ & $4 / 5$ \\
ThI & Finger abductors (little finger) & $0 / 5$ & $3 / 5$ \\
L2 & Hip flexors & $3 / 5$ & $5 / 5$ \\
L3 & Knee extensors & $2+/ 5$ & $4 / 5$ \\
L4 & Ankle dorsiflexors & I/5 & $4 / 5$ \\
L5 & Long toe extensors & $1 / 5$ & $4 / 5$ \\
S1 & Ankle plantar flexors & $0 / 5$ & $4 / 5$ \\
\hline
\end{tabular}

Notes: Muscle function grading: 0 , total paralysis; I, palpable contraction; 2 , active movement with full range of motion (ROM) with gravity eliminated; 3, active movement with full ROM against gravity; 4 , active movement with full ROM against moderate resistance; 5 , active movement with full ROM against full resistance.

EPO can be found in hypoxic neuronal tissue. It was demonstrated that the systemic application of EPO to rats has an effect of apoptosis of neuronal tissue and the functional recovery of rats with an acute SCI. ${ }^{4,5}$ The protective effect of EPO is an effect of the activation of the Nrf2 signaling pathway. ${ }^{6}$ Vitamin $\mathrm{C}$ (ascorbic acid) is an organic acid and has antioxidant abilities. It can scavenge free radicals and avoid tissue damage like secondary injury in SCI. Different studies showed that high-dose intraperitoneal administration of ascorbic acid or a vitamin C-supplemented diet can reduce secondary-injury-induced tissue necrosis and improve the functional recovery in a rat model. ${ }^{7,8}$

G-CSF is a hormone that induces the production of granulocytes. Different studies indicate that G-CSF also has neuroprotective abilities and may reduce secondary injury after acute SCI. Different clinical trials (prospective and retrospective) demonstrated that patients treated with G-CSF have a better functional recovery than people treated with methylprednisolone. ${ }^{9,10}$

Table 5 Functional grading of the key muscles 22 days postsurgery

\begin{tabular}{llll}
\hline Segment & Key muscles & Right & Left \\
\hline C5 & Elbow flexors & $5 / 5$ & $5 / 5$ \\
C6 & Wrist extensors & $2+/ 5$ & $4 / 5$ \\
C7 & Elbow extensors & $2 / 5$ & $4 / 5$ \\
C8 & Finger flexors (distal phalanx of middle finger) & $0 / 5$ & $4 / 5$ \\
ThI & Finger abductors (little finger) & $0 / 5$ & $3 / 5$ \\
L2 & Hip flexors & $3 / 5$ & $5 / 5$ \\
L3 & Knee extensors & $3 / 5$ & $4 / 5$ \\
L4 & Ankle dorsiflexors & $1 / 5$ & $4 / 5$ \\
L5 & Long toe extensors & $2 / 5$ & $4 / 5$ \\
SI & Ankle plantar flexors & $0 / 5$ & $4 / 5$ \\
\hline
\end{tabular}

Notes: Muscle function grading: 0 , total paralysis; I, palpable contraction; 2 , active movement with full range of motion (ROM) with gravity eliminated; 3 , active movement with full ROM against gravity; 4 , active movement with full ROM against moderate resistance; 5 , active movement with full ROM against full resistance. 
Table 6 Functional grading of the key muscles 31 days postsurgery

\begin{tabular}{llll}
\hline Segment & Key muscles & Right & Left \\
\hline C5 & Elbow flexors & $5 / 5$ & $5 / 5$ \\
C6 & Wrist extensors & $3 / 5$ & $5 / 5$ \\
C7 & Elbow extensors & $2 / 5$ & $4 / 5$ \\
C8 & Finger flexors (distal phalanx of middle finger) & $2 / 5$ & $4 / 5$ \\
ThI & Finger abductors (little finger) & $2 / 5$ & $3 / 5$ \\
L2 & Hip flexors & $3 / 5$ & $5 / 5$ \\
L3 & Knee extensors & $4 / 5$ & $5 / 5$ \\
L4 & Ankle dorsiflexors & $3 / 5$ & $5 / 5$ \\
L5 & Long toe extensors & $4 / 5$ & $4 / 5$ \\
S1 & Ankle plantar flexors & $3 / 5$ & $4 / 5$ \\
\hline
\end{tabular}

Notes: Muscle function grading: 0 , total paralysis; I, palpable contraction; 2, active movement with full range of motion (ROM) with gravity eliminated; 3, active movement with full ROM against gravity; 4, active movement with full ROM against moderate resistance; 5 , active movement with full ROM against full resistance.

The intention behind the therapy with these three substances was to avoid a second injury through different pathways. In 2009, most of these were not understood very well and there were no data of any human treated with them against SCI. First animal studies demonstrated that there is a positive effect onto functional recovery as well as protection of neuronal structures. ${ }^{11-14}$ Recently, Li et al ${ }^{11}$ demonstrated that SCIs are associated with chronic poor blood flow and lack of oxygen to the neuronal structure in the spinal cord. They improved blood flow and oxygenation to the neuronal networks below the injury as well as blocking aromatic 1-amino acid decarboxylase (AADC) enzyme and recovered more function in SCIs. They discovered that the capillaries were excessively constricted by pericytes after SCI. Therefore, they decided to block the AADC to improve blood flow to the neuronal networks. Kadoya et al transplanted specialized embryonic stem cells into the severed spinal cords in rat models and found extensive regeneration of the severed nerve fibers, with quite improvement in their ability

Table 7 Functional grading of the key muscles 58 days postsurgery

\begin{tabular}{llll}
\hline Segment & Key muscles & Right & Left \\
\hline C5 & Elbow flexors & $5 / 5$ & $5 / 5$ \\
C6 & Wrist extensors & $3 / 5$ & $5 / 5$ \\
C7 & Elbow extensors & $2+/ 5$ & $5 / 5$ \\
C8 & Finger flexors (distal phalanx of middle finger) & $4 / 5$ & $5 / 5$ \\
ThI & Finger abductors (little finger) & $3 / 5$ & $5 / 5$ \\
L2 & Hip flexors & $3+/ 5$ & $5 / 5$ \\
L3 & Knee extensors & $4 / 5$ & $5 / 5$ \\
L4 & Ankle dorsiflexors & $3 / 5$ & $5 / 5$ \\
L5 & Long toe extensors & $4 / 5$ & $4 / 5$ \\
S1 & Ankle plantar flexors & $4 / 5$ & $4 / 5$ \\
\hline
\end{tabular}

Notes: Muscle function grading: 0 , total paralysis; I, palpable contraction; 2 , active movement with full range of motion (ROM) with gravity eliminated; 3 , active movement with full ROM against gravity; 4 , active movement with full ROM against moderate resistance; 5 , active movement with full ROM against full resistance.
Table 8 Functional grading of the key muscles 92 days postsurgery

\begin{tabular}{llll}
\hline Segment & Key muscles & Right & Left \\
\hline C5 & Elbow flexors & $5 / 5$ & $5 / 5$ \\
C6 & Wrist extensors & $4 / 5$ & $5 / 5$ \\
C7 & Elbow extensors & $3 / 5$ & $5 / 5$ \\
C8 & Finger flexors (distal phalanx of middle finger) & $4 / 5$ & $5 / 5$ \\
ThI & Finger abductors (little finger) & $3 / 5$ & $5 / 5$ \\
L2 & Hip flexors & $3+/ 5$ & $5 / 5$ \\
L3 & Knee extensors & $4 / 5$ & $5 / 5$ \\
L4 & Ankle dorsiflexors & $3 / 5$ & $5 / 5$ \\
L5 & Long toe extensors & $4 / 5$ & $4 / 5$ \\
S1 & Ankle plantar flexors & $4 / 5$ & $4 / 5$ \\
\hline
\end{tabular}

Notes: Muscle function grading: 0, total paralysis; I, palpable contraction; 2, active movement with full range of motion (ROM) with gravity eliminated; 3, active movement with full ROM against gravity; 4 , active movement with full ROM against moderate resistance; 5 , active movement with full ROM against full resistance.

to move their forelimbs. ${ }^{12}$ SCIs create more complications such as neurogenic bladder dysfunction and central neuropathic pain in addition to paralysis and numbness. Fandel et al transplanted human embryonic stem cell-derived interneuron precursors in the spinal cords 2 weeks after injury in an injured mouse spinal cord. Those implanted cells were integrated and differentiate into human GABAergic neurons to relieve injury-related symptoms, such as neurogenic bladder dysfunction and central neuropathic pain. ${ }^{13}$ Watzlawick et al conducted a literature research of databases of 49 animal studies researchers for the regenerative potential of olfactory ensheathing cells (OECs) into the site of damage of SCI. ${ }^{14}$ They justified OECs as a cellular substrate to develop and optimize minimally invasive and safe cellular transplantation for treatment of SCI, which could lead to clinical implication into state-of-the-art Phase I/II clinical trial design studies for future human SCI.

SCI is a rare disease with an incidence about 40 cases per million population in the USA. The most common reasons are traffic accidents, falls, violence and sports..$^{15}$ Our patient presented with an incomplete tetraparesis as a result of an SCI after the accident. It was not possible to treat him with steroids because he was out of the therapeutic time period of 8 hours when he presented to the hospital. The main problem of SCIs is the secondary injury caused by inflammation and swelling of the spinal cord. To avoid this, the patient was experimentally treated with EPO intrathecal and EPO, G-CSF and vitamin C subcutaneous after his initial spinal cord relief surgery. These drugs are able to relieve this secondary reaction but were never applied for this indication in human before. Figure 2 shows the MRI image after surgery based on three patterns of MRI in SCI patients (hemorrhage, edema and contusion) correlating them with the neurological status at the time of discharge $e^{16,17}$ 

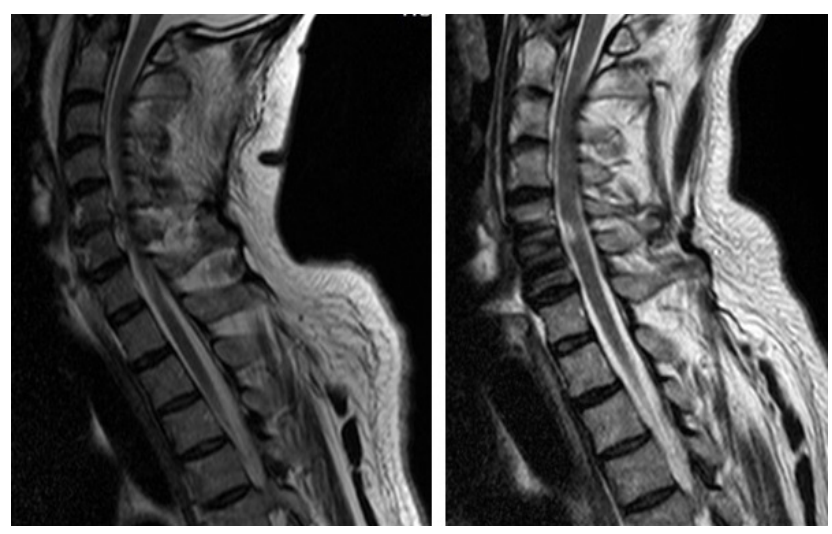

Figure 2 Magnetic resonance images of the spinal cord before (left) and after (right) initial surgical therapy.

including other patterns of cord transection ${ }^{17,18}$ and spinal cord compression ${ }^{19}$ in order to get a good prognostic value for neurological recovery and functional ambulation. We observed the significant recovery of neurological damage such as cord hemorrhage, swelling, soft tissue and ligament injury, blood clots or herniated disc in MRI spinal cord as compared to MRI spinal cord in Figure 2. The muscle function of the patient started to increase within the first 30 days, which seems to be normal in patients with incomplete paresis and spinal cord lesions. It is noteworthy that the patient almost completely recovered within the first 90 days after the injury. Only his bladder function was still affected from a functional disorder. This suggests that the experimental drug therapy can avoid secondary injury and supports the healing processes (eg, by induction of angiogenesis). This therapy may be considered in patients with SCI without any other therapeutic option.

\section{Conclusion}

SCI is a rare, often accidental trauma that has a significant effect on the life and the welfare of a patient. Patients recover mostly in the first 90 days after the injury but often have motor or sensory disorders. CT and MRI are the best ways to diagnose a SCI but there is also SCIWORA. It is necessary to treat the primary injury as well as avoid secondary injury due to inflammation and edema. G-CSF, EPO and vitamin $\mathrm{C}$ can be applied intrathecally/subcutaneously to avoid secondary injury and increase the quality of life after the injury.

\section{Acknowledgment}

Written informed consent has been provided by the patient to have the case details and any accompanying images published.

\section{Author contributions}

All authors contributed toward data analysis, drafting and revising the paper and agree to be accountable for all aspects of the work.

\section{Disclosure}

The authors report no conflicts of interest in this work.

\section{References}

1. La Rosa G, Conti A, Cardali S, Cacciola F, Tomasello F. Does early decompression improve neurological outcome of spinal cord injured patients? Appraisal of the literature using a meta-analytical approach. Spinal Cord. 2004;42(9):503-512.

2. Bracken MB, Shepard MJ, Holford TR, et al. Administration of methylprednisolone for 24 or 48 hours or tirilazad mesylate for 48 hours in the treatment of acute spinal cord injury. Results of the Third National Acute Spinal Cord Injury Randomized Controlled Trial. National Acute Spinal Cord Injury Study. JAMA. 1997;277(20):1597-1604.

3. Hall ED, Springer JE. Neuroprotection and acute spinal cord injury: a reappraisal. NeuroRx. 2004;1(1):80-100.

4. Kontogeorgakos VA, Voulgaris S, Korompilias AV, et al. The efficacy of erythropoietin on acute spinal cord injury. An experimental study on a rat model. Arch Orthop Trauma Surg. 2009;129(2): 189-194.

5. Sirén AL, Fratelli M, Brines M, et al. Erythropoietin prevents neuronal apoptosis after cerebral ischemia and metabolic stress. Proc Natl Acad Sci U S A. 2001;98(7):4044-4049.

6. Jin W, Ming X, Hou X, et al. Protective effects of erythropoietin in traumatic spinal cord injury by inducing the Nrf2 signaling pathway activation. J Trauma Acute Care Surg. 2014;76(5):1228-1234.

7. Yan M, Yang M, Shao W, et al. High-dose ascorbic acid administration improves functional recovery in rats with spinal cord contusion injury. Spinal Cord. 2014;52(11):803-808.

8. Katoh D, Ikata T, Katoh S, Hamada Y, Fukuzawa K. Effect of dietary vitamin $\mathrm{C}$ on compression injury of the spinal cord in a rat mutant unable to synthesize ascorbic acid and its correlation with that of vitamin $\mathrm{E}$. Spinal Cord. 1996;34(4):234-238.

9. Kamiya K, Koda M, Furuya T, et al. Neuroprotective therapy with granulocyte colony-stimulating factor in acute spinal cord injury: a comparison with high-dose methylprednisolone as a historical control. Eur Spine J. 2015;24(5):963-967.

10. Inada $\mathrm{T}$, Takahashi $\mathrm{H}$, Yamazaki $\mathrm{M}$, et al. Multicenter prospective nonrandomized controlled clinical trial to prove neurotherapeutic effects of granulocyte colony-stimulating factor for acute spinal cord injury: analyzes of follow-up cases after at least 1 year. Spine. 2014; 39(3):213-219.

11. Li Y, Lucas-Osma AM, Black S, et al. Pericytes impair capillary blood flow and motor function after chronic spinal cord injury. Nat Med. 2017;23(6):733-741.

12. Kadoya K, Lu P, Nguyen K, et al. Spinal cord reconstitution with homologous neural grafts enables robust corticospinal regeneration. Nat Med. 2016;22(5):479-487.

13. Fandel TM, Trivedi A, Nicholas CR, et al. Transplanted human stemCell-derived interneuron precursors mitigate mouse bladder dysfunction and central neuropathic pain after spinal cord injury. Cell Stem Cell. 2016;19(4):544-557.

14. Watzlawick R, Rind J, Sena ES, et al. Olfactory Ensheathing Cell Transplantation in Experimental Spinal Cord Injury: Effect size and Reporting Bias of 62 Experimental Treatments: a systematic review and meta-analysis. PLoS Biol. 2016;14(5):e1002468

15. National Spinal Cord Injury Statistical Center, Birmingham, Alabama (2013) SpinalCord Injury Facts and Figures at a Glance. Available from: https://www.nscisc.uab.edu/PublicDocuments/fact_figures_docs/ Facts\%202013.pdf. Accessed September 15, 2014. 
16. Kulkarni MV, Bondurant FJ, Rose SL, Narayana PA. 1.5 tesla magnetic resonance imaging of acute spinal trauma. Radiographics. 1988;8(6): 1059-1082.

17. Kalfas I, Wilberger J, Goldberg A, Prostko ER. Magnetic resonance imaging in acute spinal cord trauma. Neurosurgery. 1988;23(3): 295-299.
18. Sett $\mathrm{P}$, Crockard HA. The value of magnetic resonance imaging (MRI) in the follow-upmanagement of spinal injury. Paraplegia. 1991;29(6): 396-410.

19. Silberstein M, Hennessy O. Implications of focal spinal cord lesions following trauma: evaluation with magnetic resonance imaging. Paraplegia. 1993;31(3):160-167.

\section{Publish your work in this journal}

Therapeutics and Clinical Risk Management is an international, peerreviewed journal of clinical therapeutics and risk management, focusing on concise rapid reporting of clinical studies in all therapeutic areas, outcomes, safety, and programs for the effective, safe, and sustained use of medicines. This journal is indexed on PubMed Central, CAS,
EMBase, Scopus and the Elsevier Bibliographic databases. The manuscript management system is completely online and includes a very quick and fair peer-review system, which is all easy to use. Visit http://www.dovepress.com/testimonials.php to read real quotes from published authors.

Submit your manuscript here: http://www.dovepress.com/therapeutics-and-clinical-risk-management-journal 